\title{
A Mixed Mode Neuro-VLSI Chip for High Speed Applications
}

\author{
Khurram Waheed and Fathi M. Salam \\ Circuits, Systems, And Neural Networks Laboratory \\ Department of Electrical and Computer Engineering, \\ Michigan State University \\ East Lansing, MI 48824-1226
}

\begin{abstract}
This paper presents selected design and operation details of a custom integrated neural chip. This neural processing chip is designed in the recent $0.18 \mu \mathrm{m}$, single poly, six-layer Copper Interconnect Technology. The chip realizes an architecture that achieves the task of autonomous-learning execution times in micro to milli seconds. The core consists of basic building blocks of 4-quadrant multipliers, transconductance amplifiers, and active load resistances, for analog (forward-) network processing and learning modules. Super-imposed on the processing network are digital memory and control modules composed of D-Flipflops, ADC, Multiplying D/A Converter (MDAC), decoders, multiplexers and comparators for parameter (weight) storage, logical control and analog/digital conversions.
\end{abstract}

\section{Introduction}

The single Neural System-on-Chip (SoC) design impacts several domains of critical applications that include nanoscale biotechnology, automotive sensing, control and actuation, wireless communications, feature extraction and pattern matching etc. The core of the chip is a neurally inspired scalable (re-configurable) array network compatible with VLSI. The chip is endowed with tested auto-learning capability, realized in hardware. It is capable of handling 16 inputs, 16 outputs. There are separate 16 inputs for applying training patterns. More inputs include control interface, synchronization and stand-alone programmability of the chip resulting in an approximate die area of $4000 \times 6000$ $\mu \mathrm{m}^{2}$. The resulting SoC consumes approximately $1.5 \mathrm{mWatt}$ of power at a voltage supply of $1.5 \mathrm{~V}$.

The architectural forward network (and learning modules) process in analog continuous-time mode. Whereas, the (converged, steady-state, and/or programmed) weights can be stored on chip in digital representation. The local static memory solution was adopted to counter the problem of weight decay in generic analog implementations of neural networks. Further, it ensures autonomous high-speed operation for the designed SoC. The overall architectural design adopts engineering methods from adaptive networks and optimization principles $[3,4]$.

The 6-layer Copper $(\mathrm{Cu})$ interconnect, 0.18 micron-single poly process enables small feature size and high connectivity, resulting in dense die area of this highly interconnected network resulting in a compact powerful engine. Moreover, the special low resistance and low capacitance electrical properties of copper permit the design to achieve the high connectivity while still managing precise distributions of the resistive and the capacitive loads. These properties enable better prediction of performance and also limit signal time-delays along the interconnect.

The chip operates in four different modes:

- learn,

- (on-chip) store,

- program read/write, and

- process

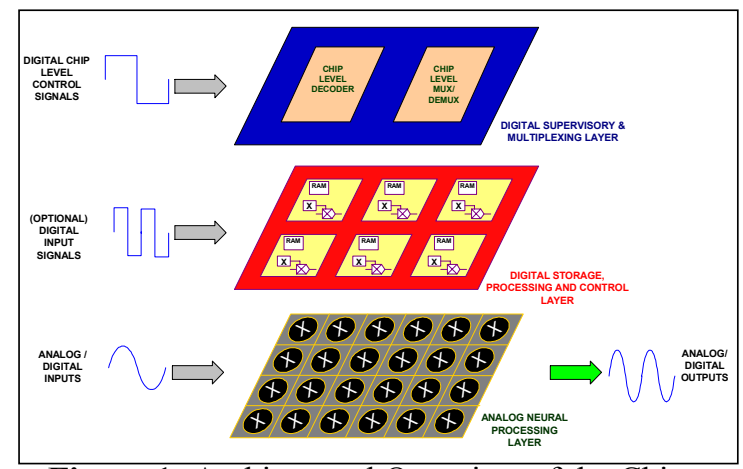

Figure 1: Architectural Overview of the Chip

These different modes selectively combine intrinsic analog and digital building blocks of the chip in a novel manner (see fig. 1), to achieve the desired tasks. More details are provided in subsequent sections. Also see [1,2,5,6].

\section{Architectural Design}

The design process comprised of consecutive stages, based on a top-down definition of the chip. A general definition of the functionality and the intended applications was created. The development of the final chip design was achieved by meticulously working through three different design stages.

- A high-level design, specifying the characteristics of the neural network to be implemented and the definition of its basic building blocks.

- A circuit level design, describing each of these blocks based on the copper technology with their corresponding simulations.

- Finally a layout level design, where the actual chip layout is created and verified 


\section{High Level Design: Modified BP Algorithm}

For implementation on VLSI, we tailored and tested the BP algorithm so that it became convenient for VLSI implementation. For more details, see $[1,5]$.

Using simulations, we have been able to verify that the network is able to converge and operate after incorporating the following changes in the modified BP Algorithm.

- Replacement of the ideal linear multiplier model by the realistic nonlinear VLSI multiplier model.

- Removal of the derivative function in all hidden layers

Note that as a result of above modifications, the training error is not zero but attains a small constant mean value. However, the update law derived in this case is still a gradient type law [3].

\section{Chip Floorplan}

The Chip is designed to be highly modular. The concept of this modularity was infused into the design right from the conception phase and realized into a granular structure of the chip as the design progressed.

The whole chip is composed of four cascaded building blocks and their interconnection. In addition, there are some global digital logic control elements (see Fig. 1). The first and the last of these blocks are routed to the padframe of the chip, as the input and the output layers of the neural engine.

\subsection{Structure of the Building Block}

The main building block of the chip comprises of a $16 \times 18$ array of building cells. The first $16 \times 1$ cells are the digital cells, while the remaining $16 \times 17$ array is formed of synaptic cells. This array of synaptic cells on the output side is padded by another column of buffers for signals to be connected to other building blocks/padframe. This stage also includes difference amplifiers used for determination of error, i.e. difference between target inputs and block outputs for tuning the local weights.

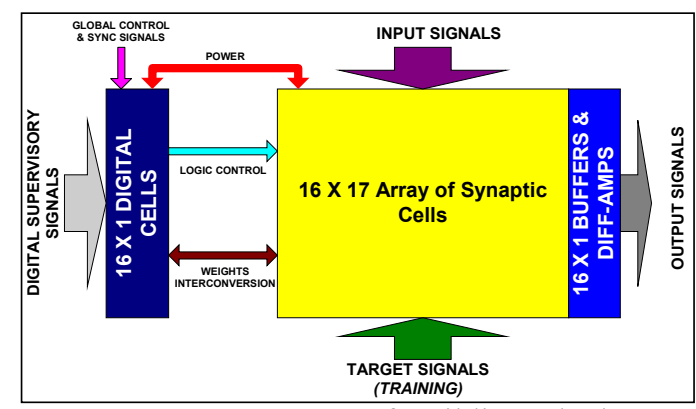

Figure 2: Structure of Building Block

An overview of this structure is provided in Fig. 2. The digital cell array receives the digital supervisory signals directly from external pins plus some global synchronization signals generated within the chip. These signals are interpreted and appropriate logic signals for the control of synaptic cells are generated. The synaptic cells for the purpose of control are addressed coded in rows and columns. This allows for a mechanism of parallel management of building block resources as well as chip level resources. Further details will be discussed in subsequent sections, also see $[1,2,6]$.

The Synaptic array can be decomposed into cascaded processing stages. Each processing stage is composed of 16 neurons built using (x17) synaptic cells and a sigmoid function. Current bus bars are used to collect output currents from each cell in a processing stage. These bus bars run horizontally and vertically for common row/column outputs. Separately designed sigmoid functions and CMOS linear resistors are used to convert these currents to voltages.

\subsection{The Synaptic Cell:}

Each synapse cell is composed of three analog Gilbert multipliers, a storage capacitor, a linear resistor, a set of transmission gates and 5 data flip-flops.

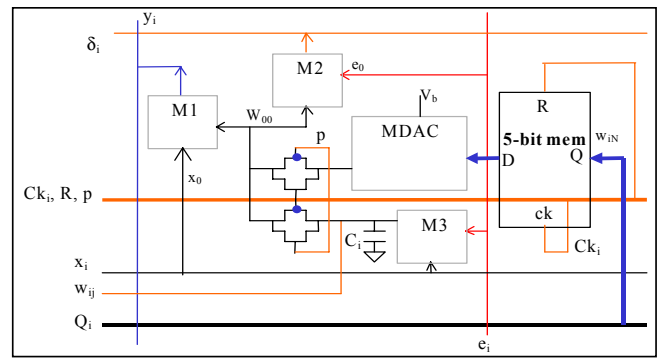

Figure 3: Synapse Cell Structure

In the learning stage, the processing multiplier M1 multiplies an input signal $\mathbf{x}_{\mathbf{i}}$ and the synapse weight $\mathbf{W}_{\mathrm{ij}}$, stored on the capacitor $\mathbf{C}_{\mathbf{i}}$ and selected by setting $\mathbf{p}$ to high, to compute the output current $\mathbf{y}_{\mathbf{i}}$. The back-propagating multiplier M2 multiplies the error signal $\mathbf{e}_{\mathbf{i}}$ with the weight $\mathbf{W}_{\mathrm{ij}}$ to calculate the feedback current component $\boldsymbol{\delta}_{\mathbf{i}}$. The weight update multiplier M3 multiplies the input signal $\mathbf{x}_{\mathbf{i}}$ and error signal $\mathbf{e}_{\mathbf{i}}$ to determine the updated weight. The current-to-voltage conversion for this multiplier is done locally using a MOS resistor. In the store mode, the locally stored weight value, using capacitor $\mathrm{C}_{\mathrm{i}}$, is converted to its digital representation. This conversion takes place in the corresponding digital cell. The converted weights are stored locally in the 5-bit memory. In the processing stage, the Multiplying DAC (MDAC) converts the stored digital bits to the equivalent weight representation. At this stage the polarity of signal $\mathbf{p}$ is adjusted so that the output of the MDAC is used as the local weight instead of the charge stored on the capacitor. The $\mathbf{R}$ signal is connected to reset of all local memory of data flip-flops, enabling the local memory to be reset independent of digital cell operation. The signal $\mathrm{Ck}_{\mathrm{i}}$ is the clock to the data flip-flops for storage of converted weights. 


\subsection{The Digital Cell}

Each digital cell contains primarily an ADC. Sharing a common ADC for all the synaptic cells in a row reduces the number of ADCs required for conversion of weight to $\sqrt{n}$. This configuration is capable of achieving satisfactory conversion time for all the weights.

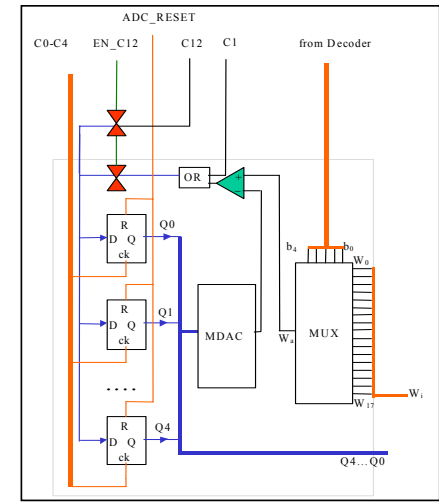

Figure 4: Digital Cell Structure

The selection of a weight in a row is controlled by chip level decoders, which do it in parallel for all rows in all the building blocks. The selected weight is compared with the MDAC output, this output is ORed with a signal C1, which can be used for weight override/clocking for the data-flipflops. The external signals C0-C4 control the clocking of the data flip-flops. The external signal $\mathbf{C 1 2}$ in conjunction with the EN_C12 signal connected to the transmission gate, provide this cell the capability to program the weights. The signal EN_C12 is generated by another set of chip-level decoders, which are supplied with the row/column selection during programming.

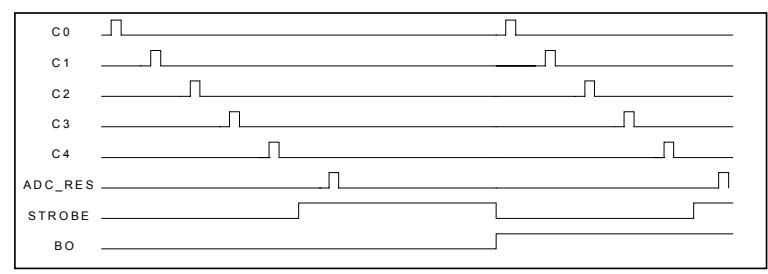

Figure 5: ADC Control Signals

The waveforms (Fig. 5) provide a synapse of the signals as required for the ADC operation. The signals STROBE and B0 belong to the associated decoder and are provided for illustration. The storage of the converted weight in the local memory is initiated by the rising edge of the STROBE signal. After which all the data flip-flops are reset using the ADC_RES signal. The transition in signal BO indicates the start of weight conversion cycle for the next in sequence synaptic cell.

\section{Design and Layout of sub-cells}

All the components for this chip were custom-designed. All the component circuits were designed on Star-Hspice using BSIM Level-49 models supplied by SRC/UMC. Avant's software SUE and METAWAVES was used for the schematic entry and waveform viewing, respectively. Initial layout of these sub-circuits was carried out in Tanner Tools but the verification and LVS were performed using Cadence Tools. Design details for some of these components are provided in $[1,5]$. In this paper we present our design of two more subcircuits that include the Multiplying MDAC [5] and the two-stage data flip-flop. All components use Vec at $1.5 \mathrm{~V}$.

\subsection{Multiplying DAC (MDAC)}
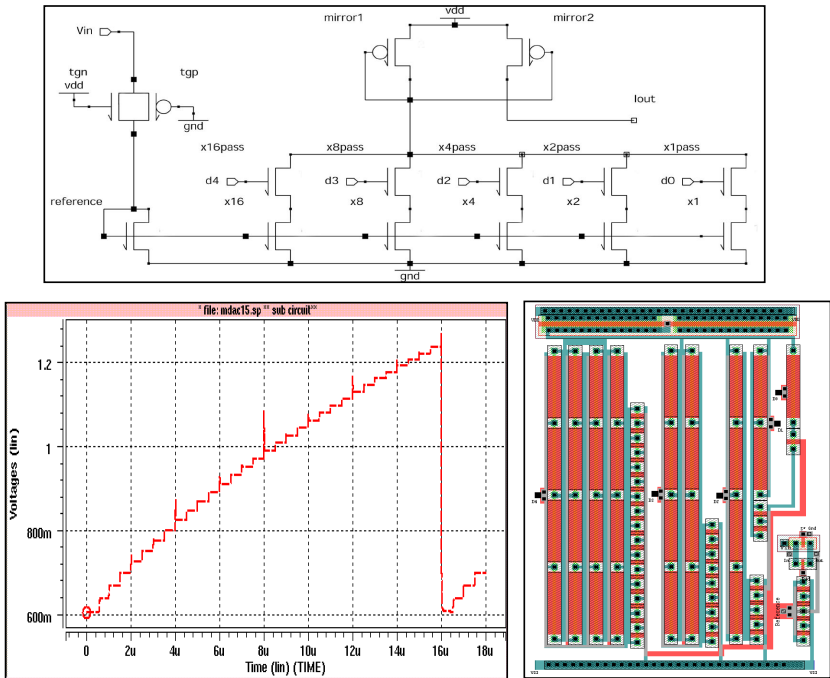

Figure 6: Multiplying DAC - Schematics, Layout and the characteristic curve

M-DAC is used for Digital to Analog conversions in the synaptic and digital cells of the designed system-on-chip. The reference current, controlled by $\mathbf{V}_{\text {in }}$, provided to the $\mathrm{M}$ DAC is $0.8 \mu \mathrm{A}$. Each successive current multiplying stage $\mathbf{i}$ is designed so that the current through it is $2^{\mathrm{i}}$ times the reference current. The input to the M-DAC is a 5-bit Digital value d4-d0. These bits enable/inhibit a multiplying stage. The total current through the multiplying stages of MDAC reflected a current mirror is used as the output. Upon conversion to voltage equivalent using a MOS linear resistor, this Digital value is mapped to an analog voltage between $0.63 \mathrm{~V}$ to $1.1 \mathrm{~V}$ at the output. Therefore the resolution of the M-DAC is $14.7 \mathrm{mV}$. The reference nmos transistors are of size $0.24 \mu \mathrm{m} / 0.4 \mu \mathrm{m}$. Other transistors are scaled accordingly. The schematic, layout and the output characteristics are shown in fig. 6.

\subsection{Data Flip-flops}

Data flip-flops are used as registers in ADCs and also as local digital memory in each synaptic cell. It is made up of four NAND and one NOT gates. This two stage structure was adopted so that the transitions in the $\mathbf{D}$ signal do not affect the flip-flop output directly. The configuration used (see Fig. 7) uses fewer numbers of transistors when compared to a typical master-slave setup, yet providing adequate performance and isolation. The maximum delay for the output of the flip-flop to go from high to low is 130 
ps and maximum delay from low to high is $70 \mathrm{ps}$. There are some minor artifacts in the output as the input changes along with the applied clock. However, the maximum glitch is less than $\pm 0.1 \mathrm{~V}$. All the nmos transistors in the design are of size $0.24 \mu \mathrm{m} / 0.20 \mu \mathrm{m}$ while all the pmos transistors are $0.8 \mu \mathrm{m} / 0.2 \mu \mathrm{m}$. The schematic, layout and the performance characteristics are provided in Fig. 7.

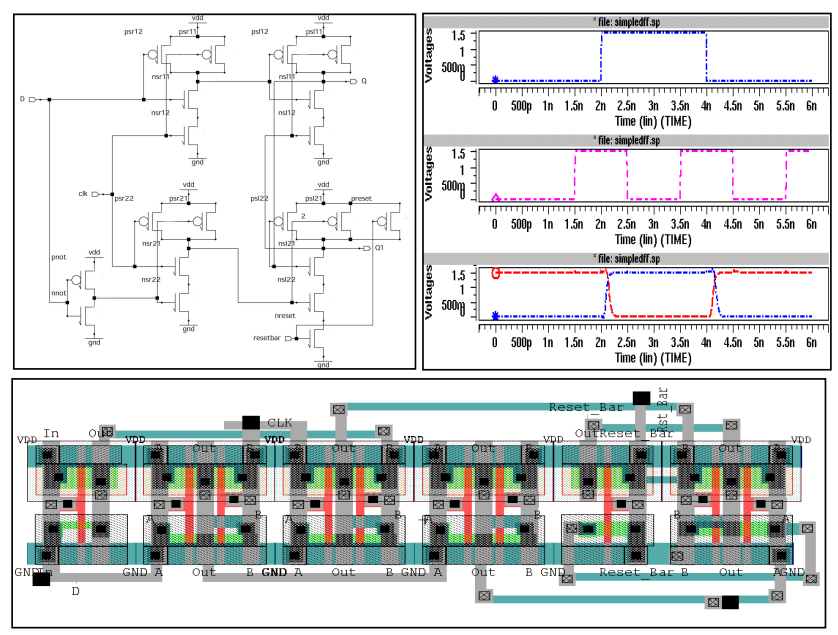

Figure 7: Data flip-flop - Schematics, Layout and the characteristic

\section{Testing Plan for the Chip}

This chip is currently under fabrication and is expected to arrive during Summer. The high pin count of the chip requires development of an automated and dedicated testbed for verification of its designed capabilities, testing various parameters and performing other applicationoriented experiments. Fig. 8 provides an overview of the interface signals required for operational testing of this SoC. The chip operates at $1.5 \mathrm{~V}$ power supply and therefore a stage of isolation/level conversion circuits have been developed for interfacing the chip with other standard digital hardware/test equipment.

The signal inputs, network outputs, training inputs to the neural network can be either analog or digital. The biases and the reference signals are used to tune the performance characteristics of the learning elements. These signals will be generated using a card from IC Tech Inc. which is primarily capable of a high number of digital i/o, in addition it can also supply a number of accurate static voltages generated using digital potentiometers and isolated lines. The digital/logic control signals B0-B4, C0-C4, SO-S3, IN0-IN3 will be generated either by the board above or National Instruments' Labview based digital i/o cards. The generation of signals from the former is done by developing programs which can generate datasets with the required timing characteristics. These timing/sampling characteristics for the board are software selectable. The board is capable of achieving high throughput rates as it can download a dataset to a microprocessor based standalone board, which then can operate on its own.

In Labview, various signal sources have been used to develop various visual instruments (VIs) to generate the interface signals, plus datasets can also be used. For the generation and the measurement of analog signals separate analog i/o boards by National Instruments will be used. However, the throughput performance of Labview under Windows platform is limited.

By design, the neural system-on-chip (nicknamed Microlearner) can accept and process signals with bandwidths greater than $100 \mathrm{MHz}$, this limit cannot be tested by the current setup. Therefore, after performing some initial validation of the chip operation, the platform for testing will be changed. The testing results for the chip will be reported in subsequent papers after its fabrication.

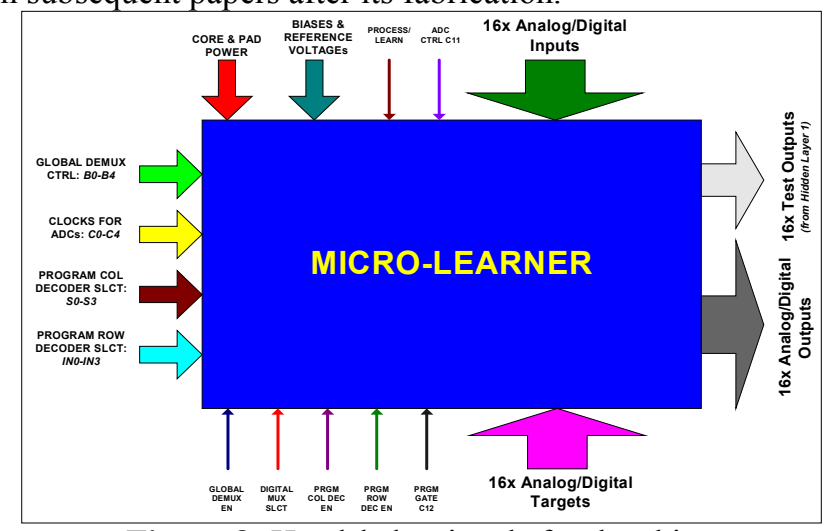

Figure 8: Handshake signals for the chip

\section{References}

[1] Khurram Waheed and Fathi M. Salam, "A Mixed Mode Self-Programming Neural System-on-Chip for Real-Time Applications"; submitted to IJCNN 2001.

[2] Khurram Waheed and Fathi M. Salam, "A Mixed-Mode Design for a Self-programming Chip For real-time estimation, prediction, and control"; Proc. Of $43^{\text {rd }}$ IEEE Midwest Symposium on Circuits and Systems, Aug 8-11, 2000, pp. 810-813

[3] Gert Cauwenberghs and M. Bayoumi, (editors) Learning on Silicon, adaptive VLSI neural systems, Kluwer Academic Publishers, July 1999.

[4] F. M. Salam, H-J. Oh, "Design of a Temporal Learning Chip for Signal Generation and Classification," Analog Integrated Circuits and Signal Processing, an international journal, Kluwer Academic Publishers, Vol. 18, No. 2/3, February 1999, pp. 229-242.

[5] MSU Team, Copper IC Design Challenge, Phase I Report, January 2000.

[6] MSU Team, Copper IC Design Challenge, Phase II Report, August 2000.

[7] Website:http://www.egr.msu.edu/annweb/cu_contest/ 